\title{
Live Demonstration: A Sensor-Processor Array Integrated Circuit for High-speed Real-time Machine Vision
}

\author{
Stephen J. Carey, David R. W. Barr, Bin Wang, Alexey Lopich, and Piotr Dudek \\ School of Electrical Engineering and Electronics \\ The University of Manchester, Manchester, M13 9PL, United Kingdom \\ e-mail: p.dudek@manchester.ac.uk
}

\begin{abstract}
A demonstration is made of the high-speed realtime image processing capabilities of the SCAMP-5 vision chip. The device provides a software-programmable 256x256 pixelparallel SIMD processor array. In the example application, the IC can determine dimensions and the location of a single object, at a sustained rate of $100,000 \mathrm{fps}$. At $30,000 \mathrm{fps}$, the chip can return the same metrics from 5 objects. This is accomplished by use of near-sensor processing which circumvents the requirement to digitise images; all processing is done "on the focal plane" and only high-level object information is transmitted off-chip as discrete address-events.
\end{abstract}

\section{INTRODUCTION}

The determination of metrics from a visual scene usually requires the readout of an imaging IC to a frame processor system, which then analyses the image. For processing modestly sized frames of $256 \times 256$ pixels at speeds of $100,000 \mathrm{fps}$ with 8-bits per pixel, data is produced at rates of 6.5 GBytes. $\mathrm{s}^{-1}$. This data presents a significant burden upon the interface and processor systems, especially if non-trivial computations need to be carried out in real-time. Typically, large and power-hungry custom parallel processing hardware would be required. In this work we demonstrate a custom vision sensor device [1]. The chip comprises an image sensor and a 256x256 processor array (one processor per image pixel), and is capable of peak performance of over 0.5 TOPS, clocking instructions into the IC at frequencies of 9,18 and $45 \mathrm{MHz}$, dependent on instruction type. Asynchronously executing binary wave-propagation instructions provide further acceleration. The I/O system allows the chip to operate in a synchronous, address-event readout mode, where only highly informative 'events', such as pixel addresses of locations of interest, resulting from executing complex image processing operations on complete image frames, are sent off-chip.

\section{DEMONSTRATION}

The demonstration (Figure 1) consists of a smart camera system built around our IC, viewing a disc rotating at 15,000 rpm - providing the objects with an effective linear speed of $130 \mathrm{kph}$. Drawn upon the disc are 5 shapes. The IC is used to locate the position of the shapes, acquiring and analyzing image frames at a rate of up to $100,000 \mathrm{fps}$. This processing rate can be sustained indefinitely, however, for visualisation purposes, the system starts acquiring images after a trigger event occurs, then captures and processes 4096 frames, returning the processed data for each frame to the host computer over a USB link. In addition to the processing results (address-events), one complete frame is returned to allow coordinate extraction verification.
Two example processing tasks are demonstrated. In the first, coordinates of one distinct shape (e.g. the "A" in Fig.1a) are returned. The spinning of the disc forces the target shape to move in a circular pattern which is visualized as a helix, with the dimensions $\{\mathrm{x}, \mathrm{y}$, time $\}$, where $\mathrm{x}$ and $\mathrm{y}$ are the coordinates of the shape, and $t$ is the time it was captured. The user can control two aspects of the system. Firstly, the rotational speed of the spinning disc. As the speed increases, the helix 'tightens', and likewise 'relaxes' as the speed is decreased. Secondly, the user can select a time at which a sample frame is returned to visually verify that the coordinate acquisition is accurate. The second program progresses much as the first but processes all the objects in the image; the host PC exploits the object size information returned by the SCAMP-5 system and plots the coordinate of the letter with the appropriate colour, depicted in Figure 1(c). The entire system is powered by the USB port. Extraction of the coordinates and sizes of all 5 letters can be performed at 30,000fps and that of a single letter at $100,000 \mathrm{fps}$.

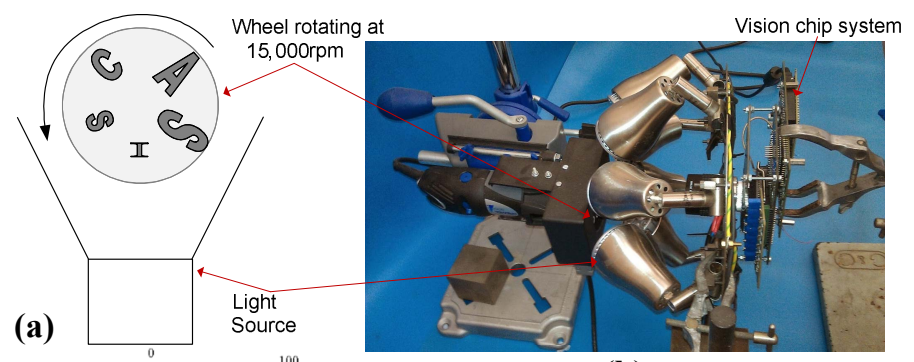

(b)

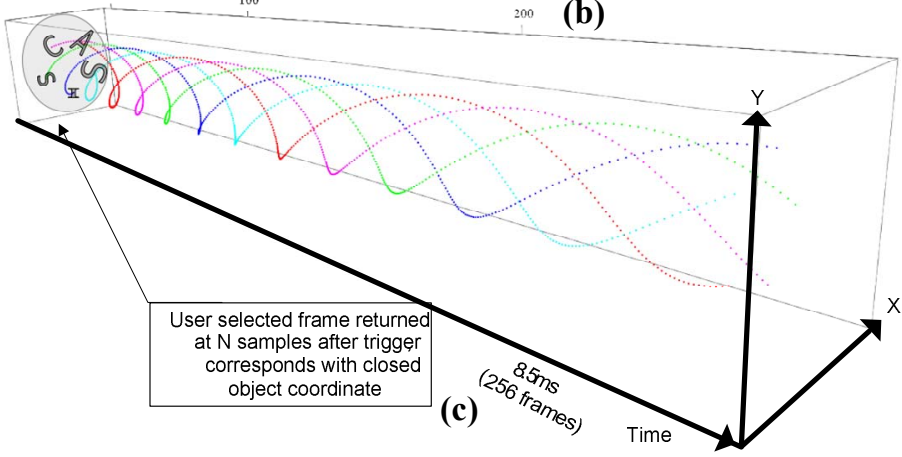

Figure 1. Demonstration setup (a) schematic view, (b) photo of the experimental setup, (c) 3D visualisation of the processing results

\section{REFERENCE}

[1] SJ Carey, A Lopich, DRW Barr, B Wang, P Dudek "A 100,000 fps vision sensor with embedded 535GOPS/W 256×256 SIMD processor array", 2013 Symposium on VLSI Circuits, C182-C183, May 2013. http://ieeexplore.ieee.org/stamp/stamp.jsp?tp=\&arnumber $=6578654$ 